\title{
Dramatic Dose Reduction in Three-Dimensional Rotational Angiography After Implementation of a Simple Dose Reduction Protocol
}

\author{
Savine C. S. Minderhoud ${ }^{1}$ D $\cdot$ Femke van der Stelt ${ }^{1} \cdot$ Mirella M. C. Molenschot $^{1} \cdot$ Michel S. Koster $^{2} \cdot$ Gregor J. Krings $^{1}$. \\ Johannes M. P. J. Breur ${ }^{1}$
}

Received: 13 February 2018 / Accepted: 28 July 2018 / Published online: 3 August 2018

(c) The Author(s) 2018

\begin{abstract}
Previously, median effective dose (ED) of $1.6 \mathrm{mSv}$ per three-dimensional rotational angiography (3DRA) has been reported. This study evaluated ED and image quality in 3DRA after implementation of a simple dose reduction protocol in pediatric catheterizations. Simple conversion factors between 3DRA ED and readily available parameters at the cathlab were determined. The dose reduction protocol consisted of frame reduction (60-30 frames/s (f/s)), active collimation of the X-ray beam, usage of a readily available low dosage program, and a pre-3DRA run check. EDs were calculated with Monte Carlo PCXMC 2.0. Three observers blindly assessed 3DRA image quality of the dose reduction and normal-dose cohort. Between October 2014 and October 2015, 84 patients (median age 4.3 years) underwent 100 3DRAs with a median ED of 0.54 mSv (0.12-2.2) using the dose reduction protocol. Median ED in the normal-dose cohort (17 3DRAs) was 1.6 mSv (1.2-4.9). Image quality in the dose reduction cohort remained excellent. Correlations between ED and dose area product (DAP) and ED and skin dose were found with a $\rho$ of 0.82 and 0.83 , respectively. ED exposure of the entire catheterization was reduced to $2.64 \mathrm{mSv}$. Introduction of a simple protocol led to $66 \%$ dose reduction in 3DRA and $79 \%$ in the entire catheterization. 3DRA image quality in this group remained excellent. In 3DRA ED correlates well with DAP and skin dose, parameters readily available at the cathlab.
\end{abstract}

Keywords Catheterization $\cdot$ Rotational angiography $\cdot$ Radiation dose reduction $\cdot$ Effective dose

\section{Abbreviations \\ 3DRA Three-dimensional rotational angiography \\ DAP Dose area product \\ ED Effective dose}

\section{Introduction}

In pediatric cardiology imaging is essential for diagnostic and interventional purposes. For this reason, patients with congenital heart disease regularly and increasingly receive radiation over the years [1]. Radiation exposure during childhood is more harmful than during adulthood. Reasons for this are the longer life span children have and the more harmful effects radiation has on developing tissue. With increased radiation exposure, children's lifetime cancer risks will increase [2-5]. Catheterizations contribute to the majority of radiation burden in patients with congenital heart disease [6].

Quite recently, three-dimensional rotational angiography (3DRA) has been added to the spectrum of image modalities. 3DRA is used for diagnostic and interventional reasons. 3DRA provides a real-time roadmap for anatomy-guided procedures and improves faster and simplified interventions with enhanced patients' safety [7]. Conversion factors enabling simple estimation of effective 
dose (ED) for standard procedures such as 3DRA acquisition have not yet been published [8]. Many studies report dose area product (DAP), a value directly provided by the imaging system $[9,10]$, but this value does not quantify the radiation's effect on patients. The effective radiation dose (ED) is the best indicator to assess the stochastic effects of radiation [3]. Furthermore, ED enables comparison between the effects of 3DRA and effects of other imaging modalities [3].

Many studies directly estimate ED from DAP [10-12]. However, the relation between DAP and ED for 3DRA has never been strongly confirmed [13]. A strong correlation will help to produce a simple formula to estimate the ED, which is more practical for daily use than the complex ED calculations.

In 2014, Peters et al. have reported a median ED of $1.6 \mathrm{mSv}$ per 3DRA in only 17 pediatric patients [14]. To limit the radiation burden, the ED should be reduced to a minimum with preservation of image quality. The ED might decrease with a few simple changes in the 3DRA protocol $[2,13,14]$.

Therefore, the aim of the present study is (1) to calculate the EDs after implementation of a simple dose reduction protocol in a larger group of patients and compare the results with Peters et al., (2) to evaluate imaging quality of this protocol, and (3) to further explore the correlation between DAP and ED [14].

\section{Materials and Methods}

\section{Study Population}

Patients were eligible for inclusion if they were $0-18$ years of age and had undergone a cardiac catheterization procedure with 3DRA acquisition at the Wilhelmina Children's Hospital between October 2014 and October 2015. The institutional review board approved this study and no informed consent was required. Retrospective analysis of medical records and catheterization data was performed. Parameters collected include age, weight, height, body surface area (BSA), cardiac diagnosis, and type of intervention (if applicable). Patients were grouped according to their initial diagnosis. Patient characteristics of this low-dose cohort 3DRA were compared with a patient group previously reported, undergoing a normal-dose 3DRA [14]. Reasons for exclusion from ED calculation were incomplete rotation, wrong positioning of the patient, and insufficient contrast. As contrast absorbs radiation, insufficient contrast leads to less radiation exposure. 3DRAs made with a central venous catheter or because of a non-cardiac diagnosis were excluded from image quality assessment.

\section{DRA Image Acquisition}

3DRAs were obtained using the Siemens Artis Zee biplane (Siemens, Forchheim, Germany) and reconstructions were transferred to the Leonardo workstation for post-processing with Syngo DynaCT Cardiac software. All procedures were performed under general anesthesia. Rapid atrial or ventricular pacing was performed in 88 of the 100 3DRAs. Pacing frequency was increased from 180/min upwards until a reduction of $50 \%$ of the systolic blood pressure was achieved. Contrast medium was administrated to the cardiac compartment prior to the region of interest meaning the right ventricle for pulmonary imaging and the left ventricle for aortic imaging. Contrast was diluted up to $60 \%$ with saline. Contrast was injected from $2 \mathrm{~mL} / \mathrm{s}$ in $3 \mathrm{~kg}$ neonates up to $16 \mathrm{~mL} / \mathrm{s}$ in $50 \mathrm{~kg}$ adolescents in case of a single injection site before start of 3DRA for $5 \mathrm{~s}$. When multiple injection sites were necessary, additional manual injections with diluted contrast were performed.

\section{Dose Reduction}

Compared to the study of Peters et al., the number of frames per second was reduced from 60 to $30 \mathrm{f} / \mathrm{s}$ [6]. In addition, patients were scanned with a tube voltage corresponding to a low-dose program (Table $\mathrm{S} 1$ ). All patients were scanned according to a protocol of one weight class lower than the patient's weight. Furthermore, a thick copper filter was used to filter out low-energy photons that can cause harm and do not contribute to the image quality. Collimation was applied with a diaphragm to protect irrelevant tissue from radiation and to prevent scattering rays from causing background haze. Before the actual run, tube current was checked to be below $100 \mathrm{~mA}$. If not, image plane was checked for metal artifacts and the tube current was automatically adjusted accordingly.

\section{Calculation of ED}

All data required for calculation were extracted from Artis Zee biplane and converted to Microsoft Excel (Microsoft, USA) with CareAnalytics (Siemens, Erlangen, Germany). Parameters describing the geometry of the X-ray tube, the radiation quality (tube current, filter material and thickness, and anode angle), and the patient (age, height, and weight) were imported in Monte Carlo program PCXMC 2.0 (STUK, Finland) from Microsoft Excel to calculate ED. The outcomes of the Monte Carlo stimulations are according to International Commission on Radiological Protection 103 (ICRP103) organ weighing factors [15, 16]. 


\section{Image Quality Assessment}

3DRA images were extracted from the Leonardo workstation and patient characteristics and cohort information were removed from the files. One experienced pediatric cardiologist and two junior researchers blindly assessed image quality independently. Pre-defined anatomical structures, necessary for diagnosis or intervention, were separately scored on a three-point scale $($ good $=3$, moderate $=2$, and poor $=1)$.

\section{Statistical Analysis}

Continuous values were expressed as median with a range, and categorical values as a number and percentage of the total. Differences between baseline characteristics were tested for significance using T-test or 2-tailed Mann Whitney test, for normally distributed and skewed continuous values, respectively. Significant differences for gender, diagnosis, and image quality were tested with a Chi-square test. $P<0.05$ was considered to be significant. A Kruskal-Wallis test was used to compare age, weight, and ED per initial diagnosis. Spearman's correlation testing and linear regression were performed to evaluate the association of ED with patient's age, height, weight, BSA, skin dose, DAP, and tube current in case of non-normally distributed variables. A Fleiss kappa was calculated to test interobserver agreement of the image quality. All statistical calculations were performed using Microsoft Excel 14.6.1.

\section{Results}

\section{Exposure Data}

Table 1 summarizes characteristics at baseline of the patients undergoing low-dose and normal-dose 3DRAs. For one patient, the height was estimated, because it was not measured during hospital admission. Analysis of baseline parameters between the two groups did not show statistically significant differences. In the low-dose group, 100 runs were performed in 84 patients. Ten patients underwent preand post-intervention 3DRA, and four patients had a second catheterization with a 3DRA. Furthermore, two patients had an additional 3DRA for evaluation of a second intervention and further evaluation of a possible vascular ring with esophageal contrast, respectively.

\section{Dose Reduction}

All 3DRAs were made with $30 \mathrm{f} / \mathrm{s}$. In 96 of the 100 3DRA, weight protocols of one weight class lower were used. Table 2 shows the radiation exposure parameters after the dose reduction.
Table 1 Baseline characteristics

\begin{tabular}{|c|c|c|}
\hline & Low dose & Normal dose [14] \\
\hline \multicolumn{3}{|l|}{ Demographic patient data } \\
\hline Number of patients & 84 & 14 \\
\hline Male, $n(\%)$ & $39(46)$ & $7(50)$ \\
\hline Age (years) & $4.29(0.0-18.8)$ & $3.79(0-16.6)$ \\
\hline Height $(\mathrm{cm})$ & $103.0(50-176)$ & $101.5(50-184)$ \\
\hline Weight (kg) & $16.15(2.4-89)$ & $14.5(3.4-57.5)$ \\
\hline $\operatorname{BSA}\left(m^{2}\right)$ & $0.69(0.19-2.10)$ & $0.65(0.23-1.68)$ \\
\hline \multicolumn{3}{|l|}{ Patient diagnosis } \\
\hline $\mathrm{PA}+\mathrm{VSD} / \mathrm{TOF}, n(\%)$ & $23(27.4)$ & $4(28.6)$ \\
\hline Aortic pathology, $n(\%)$ & $20(23.8)$ & $3(21.4)$ \\
\hline Univentricular heart, $n(\%)$ & $19(22.6)$ & $2(14.3)$ \\
\hline TGA, $n(\%)$ & $6(7.1)$ & $2(14.3)$ \\
\hline Genetic syndrome, $n(\%)$ & $8(9.5)$ & $0(0)$ \\
\hline Others, $n(\%)$ & $8(9.5)$ & $3(21.4)$ \\
\hline \multicolumn{3}{|l|}{ Procedural data } \\
\hline Number of 3DRAs & 100 & 17 \\
\hline $\begin{array}{l}\text { Interventional procedures, } \\
n(\%)\end{array}$ & $75(75)$ & $12(71)$ \\
\hline
\end{tabular}

Continuous variables are summarized as median and range, and categorical variables are reported as number of cases $(n)$ and percentage $B S A$ body surface area, $P A$ pulmonary atresia, $T G A$ transposition of the great arteries, TOF tetralogy of fallot, $V S D$ ventricular septal defect

\section{Effective Dose}

After applying the dose-reducing protocol, a mean and median ED per 3DRA of $0.67 \mathrm{mSv}( \pm 0.44 \mathrm{SD})$ and $0.54 \mathrm{mSv}$ (range 0.12-2.2), respectively, were calculated compared to a mean and median ED per 3DRA of $2.0( \pm 1.1$ $\mathrm{SD})$ and $1.6 \mathrm{mSv}(1.2-4.9)$, respectively, in the normal-dose group (Fig. 1). The reduction in ED achieved with the dose reduction protocol was highly significant $(P<0.001,95 \%$ CI 0.82-1.32). Differences in age, weight, and 3DRA ED between diagnostic groups were not significant. Only 2 of the 100 3DRAs had EDs higher than $2.0 \mathrm{mSv}$. These two patients were aged 15 and 16 years, much higher than median age of 4.3 years. Furthermore, the total procedural ED was reduced from a median total ED $12.4 \mathrm{mSv}$ in the normal-dose group to $2.64 \mathrm{mSv}$ in the low-dose group $(P<0.001,95 \%$ CI $2.18-11.28)$ (Table 2). In patients with a diagnostic catheterization, a median total ED of $0.86 \mathrm{mSv}$ was found. Also, fluoroscopy and angiography ED were reduced, while fluoroscopy and procedure time did not differ between the two groups. DAP and skin dose correlate very well with ED (Fig. 2). Spearman's correlations coefficients between DAP and ED and skin dose and ED of the 100 low-dose 3DRAs were $\rho 0.82$ and 0.83 , respectively. The correlations between DAP and ED and skin dose and ED were the strongest in patients aged $1-4.99$ years, $\rho 0.92$ 
Table 2 Technical characteristics

\begin{tabular}{lllr}
\hline & $\begin{array}{l}\text { Low dose } \\
N=100\end{array}$ & $\begin{array}{l}\text { Normal dose [14] } \\
N=17\end{array}$ & $P$ value \\
\hline Exposure parameters & & & $<0.001$ \\
$\quad$ Tube voltage 3DRA (kV) & $70(60-96)$ & $90(90-90)$ & $<0.001$ \\
Tube current 3DRA (mA) & $228(53-395)$ & $69(26-363)$ & $<0.001$ \\
Exposure time 3DRA (ms) & $465.5(333.2-1330)$ & $843.2(452.2-868)$ & $<0.001$ \\
Skin dose 3DRA (mGy) & $9(1.7-83)$ & $20,86(10,33-90,69)$ & $<0.001$ \\
DAP 3DRA (mGy·cm $\left.{ }^{2}\right)$ & $1279(150-16,987)$ & $3128(1231-17,273)$ & 0.360 \\
Procedural time (min) & $153(30-360)$ & $165(60-540)$ & 0.497 \\
Total fluoroscopy time (min) & $28(0.4-121)$ & $22(6.4-81.4)$ & $<0.001$ \\
Effective dose & & & 0.002 \\
3DRA (mSv) & $0.54(0.12-2.23)$ & $1.62(0.70-4.94)$ & 0.009 \\
Fluoroscopy (mSv) & $1.53(0.00-25.40)$ & $4.4(0.2-15.8)$ & $<0.001$ \\
Angiography (mSv) & $0.45(0.00-19.33)$ & $3.6(0-79.1)$ & \\
Total catheterization (mSv) & $2.64(0.27-28.13)$ & $12.4(2-99.9)$ & \\
Interventional group & $3.65(0.49-28.13)$ & & \\
Diagnostic group & $0.86(0.27-10.09)$ & & \\
\hline
\end{tabular}

Values represent median and range

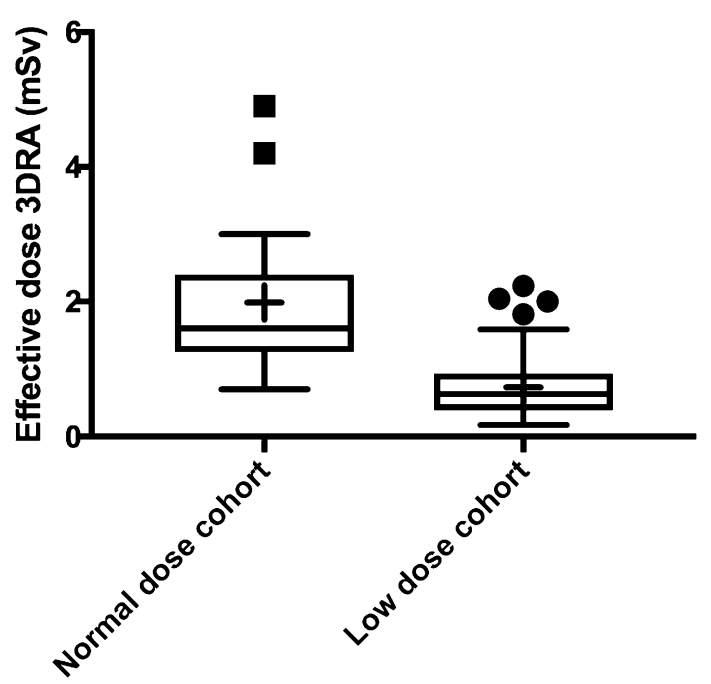

Fig. 1 Distribution of 3DRA EDs; + indicates mean value. Normal dose: $n=17$; low dose: $n=100$

and 0.93, respectively. A multiple linear regression equation was calculated to predict ED based on DAP and weight. A significant regression equation

$\mathrm{ED}=0.44-0.008 \times$ weight $(\mathrm{kg})+0.000158 \times \mathrm{DAP}\left(\mathrm{mGy} \cdot \mathrm{cm}^{2}\right)$

was found $(F(2,97)=195.435, P<0.000)$, with a $R^{2}$ of 0.80 .

\section{Image Quality}

Blind assessment of image quality was possible in 93 of the 112 3DRAs, and those were included in our analysis. The median score in both cohorts was 3; $96 \%$ of the items had a median score of good. One anatomical structure in one patient was scored poor (1), and thus overall all images had sufficient quality for clinical decision-making. Comparing the two groups, there was a significantly better image quality in the low-dose cohort. Among the three different reviewers, fair agreement was seen, with a Fleiss kappa value of 0.34 . 351 of the 390 (90\%) scored items received the same score from all three reviewers. Images of a 3-month-old patient with an aortic coarctation and 3DRA ED of $0.12 \mathrm{mSv}$ are shown in Fig. 3.

\section{Discussion}

With this study, we show that standardized use of traditional 2D angiography dose-lowering techniques leads to a $66 \%$ dose reduction in 3DRA with preserved (excellent) image quality.

Furthermore, a significant decrease in total procedural radiation dose was observed. Finally, the strong correlations between DAP and ED and skin dose and ED were confirmed and a simple formula to estimate 3DRA ED was proposed.

\section{Implications}

Annual average EDs are $3 \mathrm{mSv}$ per person of which $80 \%$ is due to natural sources such as cosmic radiation. $0.6 \mathrm{mSv}$ of annual average dose results from artificial sources such as medical exposure, atmospheric nuclear testing, and occupational exposure of which medical exposure accounts for almost $100 \%$ of the artificial ED [17]. Average annual 

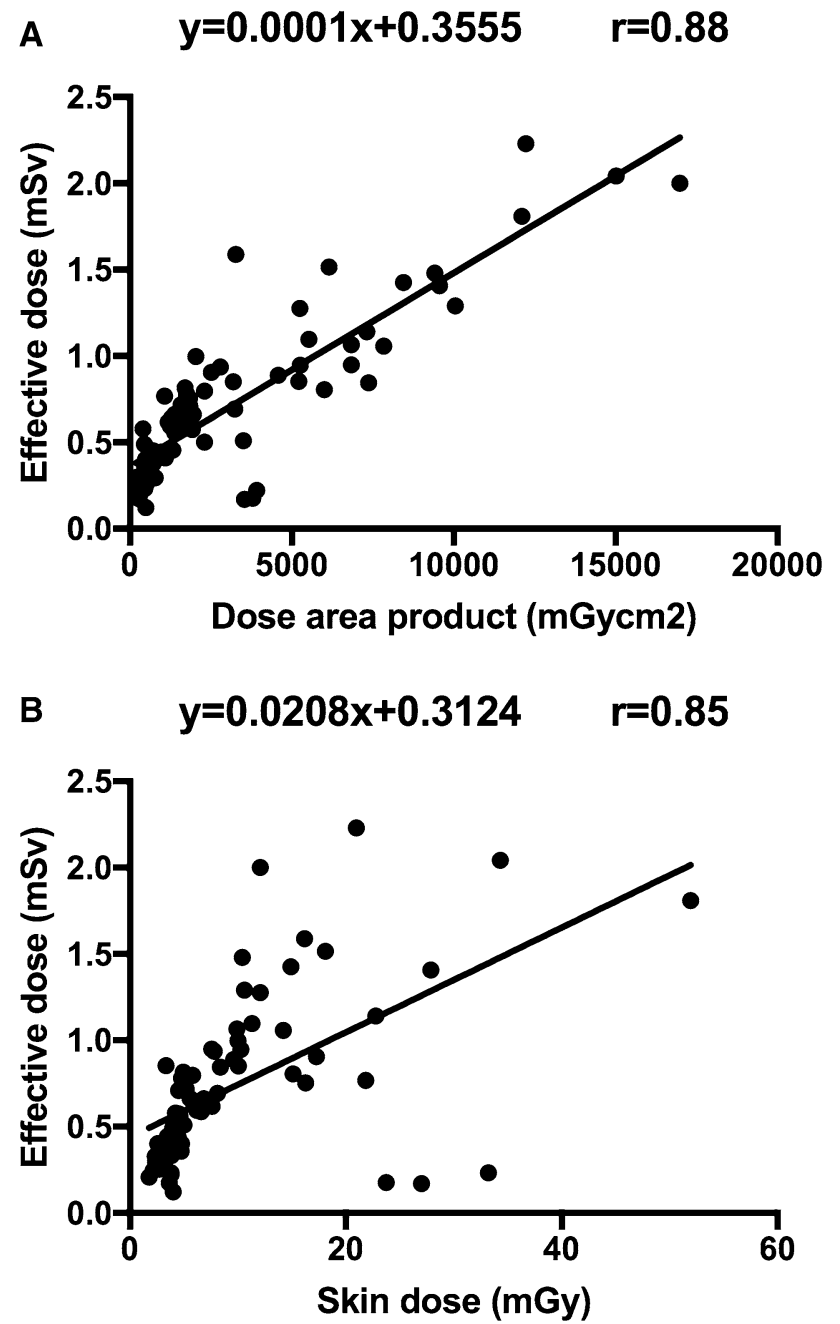

Fig. 2 a Scatterplot of DAP and ED for patients that underwent a 3DRA in the low-dose cohort-the straight lines indicate the least squared fit for the low-dose cohort. b Scatterplot of skin dose and ED for patients that underwent a 3DRA in the low-dose cohort-the straight lines indicate the least squared fit for the low-dose cohort exposure in pediatric cardiology population varies greatly. Patients with complex congenital heart disease such as a univentricular heart can have an average annual effective dose of $20 \mathrm{mSv}$ solely because of ionizing radiation-producing medical examinations [4]. As a result in patients with complex heart disease, life attributable risk of cancer was $6.5 \%$ above baseline [4]. In pediatric cardiology, cardiac catheterizations contribute to $60 \%$ of the total radiation exposure. Thus, patients included in this study are exposed to higher risks of cancer development compared to the general population. Therefore, the dose reduction of the 3DRA solely with $1 \mathrm{mSv}$ and almost $10 \mathrm{mSv}$ for the entire procedure is highly relevant.

\section{DRA and Total Catheterization EDs}

Low 3DRA EDs can be achieved using simple 2D angiography dose-lowering techniques and can, therefore, be easily applied in every cathlab. EDs of 3DRA have not been extensively calculated and reported in pediatric cardiology. Compared to other studies, our 3DRA ED is low. Table 3 provides an overview difference 3DRA ED values reported by previous studies. Reinke et al. suggested that an ED lower than $1 \mathrm{mSv}$ is possible for 3DRA in real patients [18]. Our study is first to confirm this hypothesis and to show ED can be decreased to a minimum using simple techniques without any diagnostic image quality loss.

Watson et al. compared computed tomographic angiography (CTA) with a diagnostic cardiac catheterization in pediatric patients. A median calculated ED of $0.74 \mathrm{mSv}$ and $10.8 \mathrm{mSv}$ was found for CTA and catheterization, respectively [21]. Ait-Ali et al. reported a total estimated ED for a diagnostic catheterization of $4.6 \mathrm{mSv}$ in pediatric patients [22]. Our median total ED was $0.86 \mathrm{mSv}$ in our diagnostic group and is comparable to the ED of CTA and much lower than the EDs of diagnostic catheterizations previously reported. Furthermore, in our study angiography ED was also significantly decreased. Thus, it seems

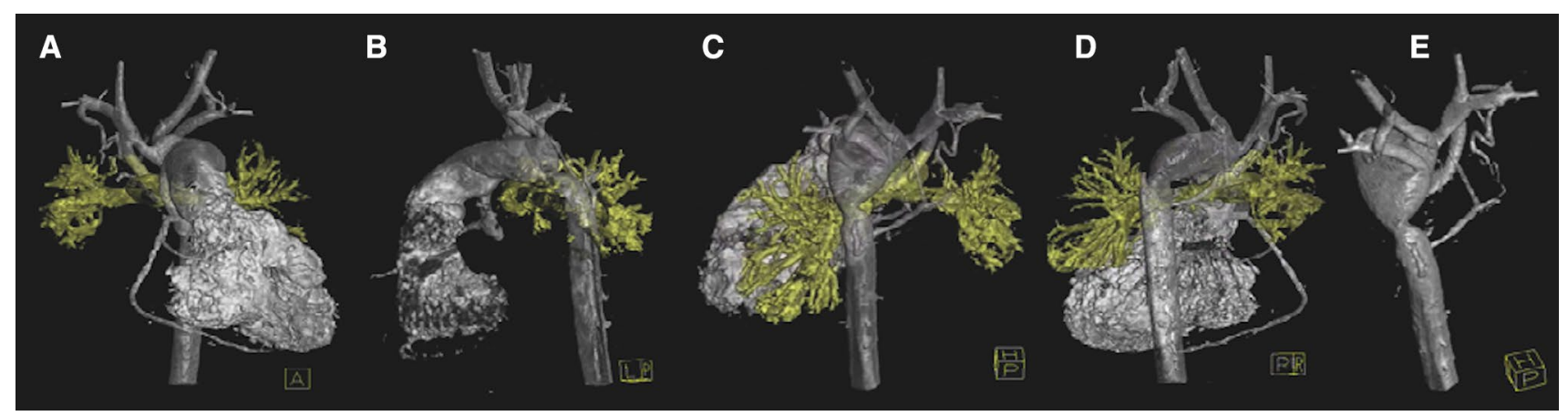

Fig. 3 3DRA with an ED of $0.12 \mathrm{mSv}$ - acquired in a 3-month-old female patient with hypoplastic left heart syndrome and aortic coarctation. The overall image quality was sufficient. a Anterior view; b lateral view; c-e posterior views, clearly showing the aortic coarctation 
Table 3 Comparison of studies on ED in 3DRA

\begin{tabular}{|c|c|c|c|c|c|}
\hline Author & $\begin{array}{l}\text { Year of } \\
\text { publica- } \\
\text { tion }\end{array}$ & Patients & Number of patients & Measure of center & $\mathrm{ED}(\mathrm{mSv})$ \\
\hline Eloot [9] & 2013 & Adults & 40 & Median & 5.7 \\
\hline Wielandts [13] & 2010 & Adults & 42 & Mean & 6.6 \\
\hline De Buck [19] & 2013 & Adults & 40 (75\% left atrium) & N/A & $\begin{array}{l}2.6 \text { (left } \\
\text { atrium } \\
\text { group) } \\
1.2 \text { (right } \\
\text { atrium } \\
\text { group) }\end{array}$ \\
\hline Haddad [10] & 2016 & $\begin{array}{l}\text { Pediatric patient; } \\
\text { age unknown }\end{array}$ & 1 & - & 1.8 \\
\hline Surendran [20] & 2017 & $\leq 2$ years & 15 & Median & 1.35 \\
\hline
\end{tabular}

N/A not available

that the excellent 3DRA image quality of this low-dose protocol reduces the need for additional angiographic imaging and the radiation burden of diagnostic catheterizations is comparable to CTA.

\section{Image Quality}

Even though $90 \%$ of the scored items received the same score, interobserver variability was fair. The small number of rating categories is the most reasonable explanation. Interestingly, image quality was better in the low-dose cohort. Enhanced operator experience and optimization of the post-processing process could explain this. Moreover, better protocols were available leading to optimal diagnostic image quality [23].

\section{Correlations with ED}

3DRA ED could be estimated with simple values as DAP and weight, which are readily available in every cathlab. Previously, Wielandts et al. found no relationship in adult patients between DAP and ED [13]. However, Peters et al. in children and Eloot et al. in adults found comparable Pearson's correlation coefficients $(r)$ of 0.87 and 0.92 , respectively [9, 14]. Recently, Haddad et al. reported Pearson's correlation coefficients ranging from 0.67 (aged $>15$ years) to 0.98 (aged $<5$ years) in 100 phantoms [10]. This confirms our finding that DAP seems to predict the ED best in the youngest patients. 3DRA is a standardized procedure and therefore DAP is likely to correlate well to ED. Correlations between ED and skin dose have not been widely published. Only from Peters et al. study a Pearson's correlation coefficient of 0.91 can be derived, which is slightly higher than our Pearson's correlation coefficient of 0.85 .

\section{Strengths and Limitations}

One of the strengths of this study is the large group of patients. This is the first study reporting 3DRA EDs from 100 patients. A fair comparison could be made with previously reported 3DRA EDs from the same center where patients underwent the same 3DRA protocol except for the above-mentioned factors. Calculation of ED was done according to the current standard [3]. A limitation of this study is the heterogenous patient group and, therefore, patients could not be directly matched to patients from the control cohort. However, the anatomical roadmap required for the procedures does not differ enormously, nor can the 3DRA procedure. This would not affect 3DRA ED values greatly. Second, ED calculations have been done based on phantom models corrected for height and weight, but not for the exact age and gender. Third, our study was based on certain protocols and equipment, which not every institution might use. This makes comparison and reproducibility harder.

\section{Conclusions}

Standardized use of traditional 2D angiography dose-lowering techniques leads to a $66 \%$ dose reduction in 3DRA with preserved image quality and a significant decrease in total procedural radiation dose. DAP and skin dose are reliable predictors of ED. The usage of the dose-reducing steps described in this study is strongly advised.

Acknowledgements The authors would like to thank Stichting Hartekind for the financial support and Rens Wientjes for his support with extracting radiation data.

Funding This study was funded by Stichting Hartekind, the Netherlands. 


\section{Compliance with Ethical Standards}

Conflict of interest The authors declare that they have no conflict of interest.

Ethical Approval This study was approved by our hospitals' review board.

Informed Consent No informed consent was required.

Open Access This article is distributed under the terms of the Creative Commons Attribution 4.0 International License (http://creativeco mmons.org/licenses/by/4.0/), which permits unrestricted use, distribution, and reproduction in any medium, provided you give appropriate credit to the original author(s) and the source, provide a link to the Creative Commons license, and indicate if changes were made.

\section{References}

1. Ladouceur VB, Lawler PR, Gurvitz M et al (2016) Exposure to low-dose ionizing radiation from cardiac procedures in patients with congenital heart disease: 15-year data from a populationbased longitudinal cohort. Circulation 133:12-20. https://doi. org/10.1161/CIRCULATIONAHA.115.019137

2. Bacher K, Bogaert E, Lapere R et al (2005) Patient-specific dose and radiation risk estimation in pediatric cardiac catheterization. Circulation 111:83-89. https://doi.org/10.1161/01.cir.0000151098 $.52656 .3 \mathrm{a}$

3. Khong PL, Ringertz H, Donoghue V et al (2013) General aspects of radiological protection in paediatric diagnostic imaging. Ann ICRP 42:21-28. https://doi.org/10.1016/j.icrp.2012.10.001

4. Johnson JN, Hornik CP, Li JS et al (2014) Cumulative radiation exposure and cancer risk estimation in children with heart disease. Circulation 130:161-167. https://doi.org/10.1161/CIRCULATIO NAHA.113.005425

5. Raissadati A, Nieminen H, Haukka J et al (2016) Late causes of death after pediatric cardiac surgery: a 60 -year population-based study. J Am Coll Cardiol 68:487-498. https://doi.org/10.1016/j. jacc.2016.05.038

6. Glatz AC, Purrington KS, Klinger A et al (2014) Cumulative exposure to medical radiation for children requiring surgery for congenital heart disease. J Pediatr 164:789-794.e10. https://doi. org/10.1016/j.jpeds.2013.10.074

7. Stenger A, Dittrich S, Glöckler M (2016) Three-dimensional rotational angiography in the pediatric Cath Lab: optimizing aortic interventions. Pediatr Cardiol 37:528-536. https://doi. org/10.1007/s00246-015-1310-6

8. Hill KD, Frush DP, Han BK et al (2017) Radiation safety in children with congenital and acquired heart disease: a scientific position statement on multimodality dose optimization from the image gently alliance. JACC Cardiovasc Imaging 10:797-818

9. Eloot L, Bacher K, Steenbeke F et al (2013) Three-dimensional rotational X-ray acquisition technique is reducing patients' cancer risk in coronary angiography. Catheter Cardiovasc Interv 82:419427. https://doi.org/10.1002/ccd.24879

10. Haddad L, Waller BR, Johnson J et al (2016) Radiation protocol for three-dimensional rotational angiography to limit procedural radiation exposure in the pediatric cardiac catheterization lab. Congenit Heart Dis 11:637-646. https://doi.org/10.1111/ chd.12356

11. Corredoira E, Vañó E, Ubeda C, Gutiérrez-Larraya F (2015) Patient doses in paediatric interventional cardiology: impact of 3D rotational angiography. J Radiol Prot 35:179-195. https://doi. org/10.1088/0952-4746/35/1/179

12. Manica JLL, Borges MS, Medeiros RF de et al (2014) A comparison of radiation dose between standard and 3D angiography in congenital heart disease. Arq Bras Cardiol 103:131-137. https ://doi.org/10.5935/abc.20140118

13. Wielandts JY, De Buck S, Ector J et al (2010) Three-dimensional cardiac rotational angiography: effective radiation dose and image quality implications. Europace 12:194-201. https://doi. org/10.1093/europace/eup394

14. Peters M, Krings G, Koster M et al (2015) Effective radiation dosage of three-dimensional rotational angiography in children. Europace 17:611-616. https://doi.org/10.1093/europace/euu207

15. Valentin RJ (2007) The 2007 Recommendations of the International Commission on Radiological Protection. Ann ICRP 37:81123. https://doi.org/10.1016/j.icrp.2006.06.001

16. Servomaa A, M. T (1998) Organ dose calculation in medical X ray examinations by the program PCXMC. Radiat Prot Dosim 80:213-219. https://doi.org/10.1093/oxfordjournals.rpd.a032509

17. UNSCEAR (2010) Sources and effects of ionizing radiation: sources Annex B. Exposures of the public and workers from various sources of radiation

18. Reinke G, Halbfaß J, Dittrich S et al (2013) Three-dimensional rotational angiography in congenital heart disease: estimation of radiation exposure. Open J Radiol 3:124-129. https://doi. org/10.4236/ojrad.2013.33020

19. De Buck S, Alzand BSN, Wielandts JY et al (2013) Cardiac threedimensional rotational angiography can be performed with low radiation dose while preserving image quality. Europace 15:17181724. https://doi.org/10.1093/europace/eut140

20. Surendran S, Waller BR, Elijovich L et al (2017) Use of 3-D digital subtraction rotational angiography during cardiac catheterization of infants and adults with congenital heart diseases. Catheter Cardiovasc Interv 90:618-625. https://doi.org/10.1002/ccd.27180

21. Watson TG, Mah E, Joseph Schoepf U et al (2013) Effective radiation dose in computed tomographic angiography of the chest and diagnostic cardiac catheterization in pediatric patients. Pediatr Cardiol 34:518-524. https://doi.org/10.1007/s00246-012-0486-2

22. Ait-Ali L, Andreassi MG, Foffa I et al (2010) Cumulative patient effective dose and acute radiation-induced chromosomal DNA damage in children with congenital heart disease. Heart 96:269 274. https://doi.org/10.1136/hrt.2008.160309

23. Starmans NLP, Krings GJ, Molenschot MMC et al (2016) Threedimensional rotational angiography in children with an aortic coarctation. Neth Heart J 24:666-674. https://doi.org/10.1007/ s12471-016-0899-2 